\title{
Balmer and Soft X-ray Emission from Solar and Stellar Flares
}

\author{
C.J. Butler
}

Armagh Observatory, $N$. Ireland

Summary: Integrated soft X-ray (8-12A) fluxes for solar flares have been scaled to the equivalent EXOSAT fluxes using spectra obtained from a variety of rocket-based experiments. The data show good agreement with the soft X-ray - $\mathrm{H} \gamma$ correlation established by Butler et al. (1988) for stellar flares and confirm the basic similarity, in this respect, of flares on the Sun to those on dMe stars.

\section{Introduction}

It was recently shown by Butler, Rodono and Foing (1988), (henceforth Paper I), that there exists a well defined linear correlation between the integrated energy emitted in $\mathrm{H} \gamma$ and soft $\mathrm{X}$-rays in stellar flares. Their data also included a single solar flare for which the equivalent soft X-ray flux in the EXOSAT CMA passband (0.04-2 KeV) had been computed by Doyle et al. (1988). This single point appeared to confirm that the relationship established for stellar flares was also appropriate to flares on the Sun.

Nearly two decades ago a similar correlation had been proposed by Thomas and Teske (1971), based, not on the direct, simultaneous, observations of individual flares in Balmer lines and soft X-rays as used in Paper I, but by observations of solar flares for which the $\mathrm{H}$-alpha class was known. In their work, the $\mathrm{H} \alpha$ and $\mathrm{X}$-ray observations were made at different times, but could be related by a common, photographically determined, $\mathrm{H} \alpha$ class. It is difficult to compare their plot for solar flares directly with those for stellar flares due to the fact that their soft X-ray fluxes were measured by OSO III over a wavelength range 8-12 A which only partly overlaps the much broader bandwidths of the EXOSAT CMA (6-280 A) and the EINSTEIN IPC (3-62 A) with which the stellar flares were observed.

\section{Scaling Factors for Solar Flares}

The question arises as to whether the soft $\mathrm{X}$-rays over the 8-12 $\mathrm{A}$ region can be scaled to determine an equivalent flux over the 6-280 A wavelength region used for stellar flares. Regrettably, there is a relative scarcity of data in the soft X-ray - EUV region for solar flares, and much that exists is from the early days of rocket experiments. We have, in fact, not been able to find a single spectrum that covers the entire region, either for a solar flare or for the quiet Sun. The problem is further complicated by the large number of emission 
lines present in the soft X-ray/EUV region. Nevertheless, fluxes have been published for several spectra, which could allow us to build up scaling factors from one wavelength region to another, and thereby determine an approximate overall scaling factor to relate $\mathrm{F}_{8-12}$ to $F_{6-280}$. The following spectra were used in the determination of these scaling factors:

(i) A flare spectrum from 6-25 A by Neupert et al. (1973)

(ii) A flare spectrum obtained simultaneously in two wavelength ranges: 15-50 A and 139-430 A by Freeman and Jones (1970)

(iii) A spectrum of the quiet Sun from 15-90 A by Freeman and Jones (1970)

(iv) A spectrum of the quiet Sun from 30-130 A by Manson (1967).

The wavelength regions where there is known to be strong emission, e.g. 5-50 A and 139-280 $A$, are covered adequately by the three flare spectra (i) and (ii), however for the region from 50-139 A we have not been able to find a suitable calibrated flare spectrum. Fortunately the flux in this region is of the order of a factor 3 or so less than the combined flux in regions 6-50 $A$ and 139-280 A where the strongest lines occur. Whilst there are undoubted differences in the fluxes of emission lines during flare as compared to quiet periods we have, in the absence of flare data for the spectral region 50-139 A, been forced to assume that the ratio of fluxes in the appropriate wavelength bands will be approximately the same during flare and quiet periods. Also the fluxes listed in the above publications are often only for emission lines and not for continua. Whereas the emission line fluxes, in general, dominate the energy in the 6-280 A range, the continuum can sometimes represent a reasonable fraction of the total flux. Here we have assumed that the continuum will scale roughly as the emission lines flux, an assumption which is probably reasonable given that much of the continuum appears to be due to unresolved lines. In this case the relative flux over different wavelength ranges will be unaffected. With these provisos in mind we give in Table 1 values for the flux in units of $\mathrm{F}_{8-12}$.

Table 1: Soft X-ray flux in units of $\mathrm{F}_{8-12}$

$\begin{array}{cccc}\mathrm{F}_{6-25} & \mathrm{~F}_{25-90} & \mathrm{~F}_{90-130} & \mathrm{~F}_{139-280} \\ 4.0 & 4.8 & 0.9 & 5.5\end{array}$

It will be noticed that the region 130-139 A is not included; unfortunately we have not been able to find a calibrated spectrum for this range. Assuming that the contribution from this range is negligible, the total flux in the $6-280 \mathrm{~A}$, or $0.04-2 \mathrm{KeV}$, range is found to be approximately 15 times the flux in the 8-12 A range used by Thomas and Teske (1971).

Applying the factor of 15 , the sum of the fluxes in Table 1, to convert the 8-12 A flux to the equivalent EXOSAT CMA flux, and the factor of $1 / 3$, as was used by Paper I, to convert the $\mathrm{H} \alpha$ flux to $H \gamma$ flux we may plot the solar flare data of Thomas and Teske (1971) on the same diagram as the stellar data from Paper I. In figure 1 we see that the data from solar and stellar flares are, to within the errors of measurement, in complete agreement, and that a single line will satisfy both sets of data. Thus we confirm that the solar flares obey the same, apparently linear, relationship between the Balmer and soft X-ray flux as do their stellar counterparts. 


\section{Interpretation}

As discussed in Paper I and by Haisch (1988), the high degree of correlation between the Balmer and soft $\mathrm{X}$-ray emission, is unexpected for the following reasons:

(i) The temperature regimes, in which the soft $\mathrm{X}$-rays and Balmer emission arise, are widely different; the former at $10^{6}-10^{7} \mathrm{~K}$ and the latter at $10^{4} \mathrm{~K}$.

(ii) The time-profiles (light curves) of some flares show an appreciable impulsive component in the Balmer flux, (see the flare on AD Leo by Rodono et al. 1989), whereas the soft $\mathrm{X}$-ray flux is commonly supposed to arise from the thermal or gradual phase of a flare.

(iii) The lower Balmer lines $\mathrm{H} \alpha, \mathrm{H} \beta$ and $\mathrm{H} \gamma$ etc are expected to be optically thick and therefore their integrated flux should depend on the shape of the plasma.

The relationship between the Balmer and soft X-ray emission should be taken into consideration in forming models of flares and would seem to mitigate against some that have been proposed. For instance one model proposes that the Balmer emission arises from cooling loops that lie beneath the arcade of hot loops containing the X-ray emitting plasma. Such a picture would suggest that the Balmer emission should always reach its peak emission

Figure 1. The equivalent $\mathrm{H} \gamma$ and EXOSAT CMA flux for the solar flare data of Thomas and Teske (1971) - open cicles, plotted with the data for stellar flares in Paper I - filled symbols.

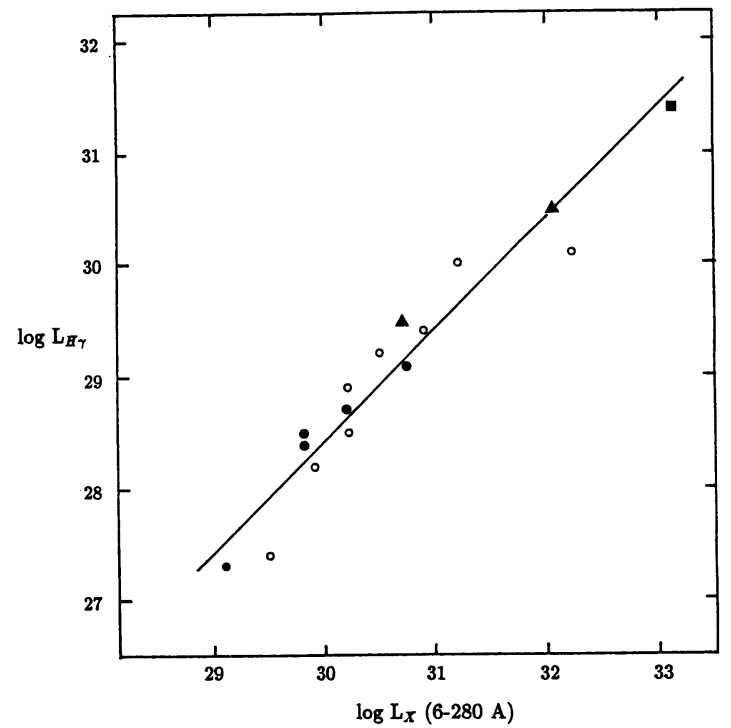

after the X-ray peak. As Haisch (1988) as pointed out, this simple progression whereby the Balmer flux always follows the soft X-ray flux does not correspond to reality; in some cases the $\mathrm{X}$-ray event precedes the Balmer emission and in some cases, vice versa. The stellar flare observed by Kahler et al. (1982) on YZ CMi is a clear example of a flare where the Balmer emission peaked earlier than the soft X-rays.

Paper I, from energy budget considerations, and assuming reasonable values for the Balmer decrement and the ratio of Balmer to Lyman flux, concluded that the total hydrogen emission was approximately two thirds of the downward-directed soft X-ray flux. However 
the EUV flux, which is also capable of exciting hydrogen, was estimated to be only of the order of half the soft X-ray flux; an estimate we now believe to be eroneous. From a comparison of the two spectra of the quiet Sun by Freeman and Jones (1970); one of the 465-794 A region, and the other of the 15-90 A region, and the various scaling factors that have already been discussed, we find that the flux in the 465-794 A region is approximately 2.6 times greater than the flux in the 6-280 A region. Thus the total EUV flux capable of exciting hydrogen may be of the order of five times greater than the soft X-ray (6-280 A) flux. In this connection we may also note the recent results of emission measure analysis by Doyle (1989) which give values of the ratio of EUV/soft X-ray flux for nine dMe stars. These ratios vary from 0.4 to 6.3 with an average value of 3.5 .

As computations by Mullan and Tarter (1977) and Cram (1982) indicated a possible efficiency of $10-20 \%$ for conversion of soft X-ray photons to hydrogen emission, it would appear that there is sufficient energy in the soft X-ray and EUV regions combined to produce the observed hydrogen flux. Nevertheless, as Haisch (1988) has pointed out, there are two fatal problems with this explanation for the correlation between Balmer and soft X-ray flux:

(i) the lack of a one-to-one relationship between soft X-ray and Balmer flux over all phases of flares - as would be predicted if the Balmer flux were to arise soley from the excitation by flare $\mathrm{X}$-ray photons,

(ii) the spatially complex patterns of $\mathrm{H} \alpha$ ribbons would not be expected if the excitation arose from a diffuse beam of photons from a high altitude coronal source.

These difficulties may well be overcome if the EUV flux can be shown to follow the time profile for the Balmer emission rather than that of the soft X-rays in solar and stellar flares.

Acknowledgement: Research at Armagh Observatory is grant-aided by the Northern Ireland Department of Education.

\section{References}

Butler, C.J., Rodono, M. and Foing, B.F.: 1988, Astron. and Astrophys. 206, L1

Cram, L.E.: 1982, Astrophys. J. 253, 768

Doyle, J.G., Butler, C.J., Callanan, P.J., Tagliaferri, G., de la Reza, R., White, N.E.,

Torres, C.A. and Quast, G.: 1988, Astron. and Astrophys. 191, 79

Doyle, J.G: 1989, Astron. and Astrophys. 214, 258

Freeman, F.F. and Jones, B.B.: 1970, Solar Phys. 15, 288

Haisch, B.M.: 1988, private comm.

Kahler, S. et al.: 1982, Astrophys. J. 252, 239

Manson, J.E.: 1967, Astrophpys. J. 147, 703

Mullan, D.J. and Tarter, C.B.: 1977, Astrophys. J. 212, 179

Neupert, W.M., Swartz, M. and Kastner, S.O.: 1973, Solar Phys. 31, 171

Rodono, M., Houdebine, E.R., Catalano, S., Foing, B.H., Butler, C.J., Scaltriti, F.,

Cutispoto, G., Gary, D.E., Gibson, D.M. and Haisch, B.M.: 1989, Poster Paper,

IAU Colloquium No 104, Stanford, Aug. 1988 - Catania Astrophys. Obs., p 53

Thomas, R.J. and Teske,R.G.: Solar Phys. 16, 431 
APPENZELLER: Can you tell from the observed Balmer decrement whether the Balmer lines are due to recombination or due to collisional excitation?

BUTLER: I think the observed Balmer decrement can be explained by collisional excitation. Some calculations on this have been made by Houdebine and by Katsova et al. However, the observations are not straight forward due to the coalescence of the higher Balmer lines and the difficulty of determining the continuum level. Also our observations only cover the blue region of the spectrum, not H-alpha or H-beta. 